\title{
EFICIÊNCIA DE ISCAS GRANULADAS À BASE DE SULFLURAMIDA E DE CLORPIRIFÓS NO CONTROLE DE Atta sexdens sexdens (HYMENOPTERA: FORMICIDAE), NO TRÓPICO ÚMIDO.
}

\author{
Adalton Pinheiro da CRUZ', José Cola ZANUNCIO², Ronald ZANETTI ${ }^{3}$, Odilávio Sá \\ GOMES ${ }^{1}$
}

\begin{abstract}
RESUMO - Este trabalho foi realizado em plantios de eucalipto urograndis (Eucalyptus urophylla $x$ Eucalyptus grandis) da Jari Celulose S.A., no município de Almeirim, Pará, no período de agosto/94 a janeiro/95. Foi testada a eficiência de uma isca granulada com sulfluramida e de três outras com clorpirifós, em três dosagens cada por metro quadrado de formigueiro, no controle de Atta sexdens sexdens (Hymenoptera: Formicidae). Os resultados foram comparados com os de uma isca à base de dodecacloro $(0,45 \%)$ a 10 gramas por metro quadrado de formigueiro. $A$ isca granulada com sulfluramida a 6,8 e 10 gramas por metro quadrado de formigueiro apresentou $77,78 \%, 100,00 \%$ e $88,90 \%$ de controle, respectivamente, assemelhando-se à isca a base de dodecacloro que apresentou $100,00 \%$ de formigueiros mortos. As outras iscas, em qualquer dosagem, apresentaram eficiência abaixo de $45,00 \%$.
\end{abstract}

Palavras-chave: Atta sexdens sexdens, formiga cortadeira, iscas granuladas, saûva-limão, sulfluramida, clorpirifós.

Efficiency of Sulfluramid and Chlorpirifos Baits Against Atta sexdens sexdens (Hymenoptera: Formicidae) in Tropical Humid Region.

ABSTRACT - This research was developed in hibrid urograndis (Eucalyptus urophylla $x$ Eucalyptus grandis) plantation belonging to Jari Celulose S.A., in Pará State, Brazil, from August 1994 to January 1995 . The objective was to test one granulated bait with sulfluramid and three others with chlorpirifos in three dosages compared to 10 grams of dodecachlor $(0.45 \%)$ bait per square meter of ant nest against Atta sexdens sexdens (Hymenoptera: Formicidae). The bait with sulfluramid showed $77.78 \% ; 100.00 \%$; and $88.90 \%$ efficiency against $A$. sexdens sexdens at 6,8 , and 10 grams while the dodecachlor bait showed $100.00 \%$ control. The other baits and dosages showed efficiency below $45.00 \%$.

Key-words: Atta sexdens sexdens, leaf cutting ants, granulated baits, sulfluramid, chlorpirifos.

\section{INTRODUÇÃO}

As formigas cortadeiras, pertencentes à tribo Attini, representam uma das pragas mais sérias na região neotropical, sendo responsáveis por grandes prejuízos aos setores agrícola e florestal (MARICONI, 1970). Segundo VILELA (1986) esses insetos respondem por mais de $75 \%$ do custo e do tempo total gasto para o controle de pragas em reflorestamentos.

Neste contexto, vários métodos de controle destes insetos foram desenvolvidose aperfeiçoados incluindo a termonebulização (COUT0 et al., 1977; SANTOS et al., 1979) e as iscas granuladas, estas consideradas o método mais eficiente, econômico e prático (ZANUNCIO et al., 1980).

Jari Celulose S.A. 68240-000, Monte Dourado, Pará

DBA/UFV. 36571-000, Viçosa, Minas Gerais.

3 DEF/UFV. 36571-000, Viçosa, Minas Gerais. 
A sulfluramida foi lançada recentemente no mercado de iscas como substituto do dodecacloro (ZANUNCIO $e t$ al., 1993e). Este produto tem sido utilizado nos EUA para o controle de Atta texana (CAMERON, 1990), de Solenopsis invicta e de formigas em residências (VANDER MEER et al., 1985; 1986) e apresenta baixa toxicidade ao homem e baixo impacto ambiental, apresentando uma meia vida muito menor que a da molécula de dodecacloro. Além destas, outros produtos químicos, como o clorpirifós, o carbosulfan e o terbujós, e naturais, como sementes de girassol e capim braquiarão, estão ou poderão vir a ser estudados como princípio ativo de iscas formicidas (DELLA LUCIA \& VILELA, 1993).

No B rasil a isca Mirex-S (sulfluramida $0,3 \%$ ) mostrou-se altamente eficiente para o controle de Atta laevigata (ZANUNCIO et al., 1992; ZANUNCIO et al., 1993d; FORTI et al., 1993); Atta bisphaerica (ZANUNCIO et al., 1993a); Atta capiguara e Atta sexdens rubropilosa (FORTI et al., 1993; ZANUNCIO et al., 1993d; LARANJEIRO \& ZANUNCIO, 1994); Acromyrmex crassispinus (ZANUNCIO et al., 1993c); Acromyrmex aspersus (CAETANO et al., 1993) e Acromyrmex subterraneus molestans (ZANUNCIO et al., 1993b). Porém, nenhum trabalho foi realizado para testar a eficiência desta isca e de outras à base de clorpirifós para A. sexdens sexdens, outra espécie de formiga cortadeira de grande importância na região Norte do Brasil.

Neste sentido objetivou-se testar a eficiência de uma isca granulada com sulfluramida e de três outras com clorpirifós, em diferentes dosagens, em comparação com uma isca à base de dodecacloro $(0,45 \%)$, para o controle da formiga cortadeira $A$. sexdens sexdens.

\section{MATERIAL E MÉTODOS}

Este trabalho foi desenvolvido em plantios de eucalipto urograndis (Eucalyptus urophylla $\times$ Eucalyptus grandis) da Jari Celulose S.A., no município de Almeirim, Pará, de agosto de 1994 a janeiro de 1995. Os tratamentos consistiram na aplicação de iscas granuladas em diferentes dosagens por metro quadrado de formigueiro (Tab. 1).

Foram marcados nove formigueiros, para cada tratamento. A avaliação de carregamento foi feita 24 horas após a aplicação das iscas e as de atividade dos formigueiros após $5,15,30,60,90$ e120 dias. Nesta última avaliação os formigueiros foram abertos com retroescavadeira e sondados com sonda JP quando se avaliou a mortalidade dos mesmos.

A quantidade de isca aplicada, em cada tratamento, foi baseada na área total de terra solta após a medição de cada formigueiro, e colocada em uma única vez em olheiros de abastecimento.

\section{RESULTADOS E DISCUSSÃO}

De modo geral, o carregamento da isca com sulfluramida foi semelhante àquele apresentado pela isca à base de dodecacloro (Tab. 2), demostrando a sua boa aceitação por operárias de A. sexdens sexdens, enquanto as demais iscas apresentaram baixa aceitação. As iscas à base de clorpirifós apresentaram reduzida percentagem de transporte e maior 
Tabela 1. Tratamentos utilizados para o controle de Atta sexdens sexdens (Hymenoptera: Formicidae). Almeirim, Pará. Agosto de 1994 a janeiro de 1995.

\begin{tabular}{llcccc}
\hline Tratamento & \multicolumn{1}{c}{ Isca Granulada } & $\begin{array}{c}\text { Dosagem } \\
\left(\mathrm{g} / \mathrm{m}^{2}\right)\end{array}$ & $\begin{array}{c}\text { Area Média } \\
\text { dos } \\
\text { Formigueiros } \\
\left(\mathrm{m}^{2}\right)\end{array}$ & $\begin{array}{c}\text { Número Médio } \\
\text { de Canais por } \\
\text { Formigueiro }\end{array}$ & $\begin{array}{c}\text { Número } \\
\text { Médio de } \\
\text { Canais por m }\end{array}$ \\
\hline 1 & $\begin{array}{l}\text { Neutra (sem principio } \\
\text { ativo) }\end{array}$ & 10 & 51.31 & 7.78 & 0.15 \\
2 & Dodecacioro & 10 & 47.34 & 7.44 & 0.16 \\
3 & Sulfluramida & 6 & 33.91 & 5.67 & 0.17 \\
4 & Sulfluramida & 8 & 29.42 & 3.67 & 0.12 \\
5 & Sulfluramida & 10 & 29.42 & 3.67 & 0.12 \\
6 & Clorpirifós A & 10 & 73.92 & 8.22 & 0.11 \\
7 & Clorpirifós A & 15 & 38.16 & 8.56 & 0.22 \\
8 & Clorpirifós A & 20 & 35.49 & 7.00 & 0.20 \\
9 & Clorpirifós B & 10 & 55.95 & 9.11 & 0.16 \\
10 & Clorpirifós B & 15 & 48.97 & 9.22 & 0.19 \\
11 & Clorpirifós B & 20 & 52.26 & 9.00 & 0.17 \\
12 & Clorpirifós C & 5 & 34.20 & 5.00 & 0.15 \\
13 & Clorpirifós C & 10 & 38.84 & 6.89 & 0.18
\end{tabular}

impacto até 30 dias, a partir do qual vários formigueiros que estavam paralisados, voltaram à apresentar atividade nas demais avaliações.

A isca com sulfluramida apresentou eficiência de $77,78 \%$, $100,00 \%$ e $88,89 \%$ para as dosagens de 6,8 e 10 gramas por metro quadrado de formigueiro, respectivamente, assemelhando-se à da isca à base de dodecacloro que apresentou $100,00 \%$ de eficiência (Tab. 2). Esses dados corroboram com aqueles de ZANUNCIO et al. $(1992 ; 1993 \mathrm{e})$ quando avaliaram a eficiência desta isca contra A. laevigata e A. bisphaerica.

As demais iscas, em todas as dosagens, apresentaram eficiência abaixo de $45,00 \%$, não sendo, portanto, indicadas para o controle de $A$. sexdens sexdens na região. Quando se considerou apenas os formigueiros que transportaram mais de $50,00 \%$ da isca aplicada, verificou-se que as iscas com sulfluramida à base de dodecacloro permaneciam com eficiência semelhante, enquanto duas iscas com clorpirifós passaram a apresentar eficiência acima de $50,00 \%$, o que é, ainda, muito baixa. Verifica-se, portanto, que as iscas com clorpirifós, nas dosagens testadas, foram ineficientes no controle de $A$. sexdens sexdens. Ao contrário, a isca com sulfluramida, nas dosagens de 6 , $10 \mathrm{e}$, principalmente, 8 gramas por metro quadrado de formigueiro, foi eficiente no controle desta formiga cortadeira e deve ser recomendada para essa finalidade.

A abertura dos formigueiros aos 120 dias mostrou pouca variação em relação à avaliação feita aos 90 dias, indicando que a maioria dos formigueiros sem movimentação externa nesta data estavam mortos (Tab. 2). Portanto a avaliação da eficiência de iscas granuladas no controle operacional de $A$. sexdens sexdens pode ser realizada aos 90 dias 
Tabela 2. Percentagem de aceitação das iscas e da paralisação de formigueiros e eficiência por tratamento e por dia de avaliação das iscas utilizadas para o controle de Atta sexdens sexdens (Hymenoptera: Formicidae). Almeirim, Pará. Agosto de 1994 a janeiro de 1995.

\begin{tabular}{lcccccccc} 
& Aceitação & \multicolumn{5}{c}{ Paralisação $(\%)$} & \multicolumn{3}{c}{ Eficiência (\%) } \\
\hline Tratamento (Isca) & Média (\%) & Dia 5 & Dia 15 & Dia 30 & Dia 60 & Dia 90 & Dia 120 \\
\hline Neutra(10) & 100,00 & 0,00 & 11,11 & 11,11 & 33,33 & 44,44 & 0,00 \\
Dodecacloro (10) & 94,44 & 11,11 & 33,33 & 33,33 & 100,00 & 100,00 & 100,00 \\
Sulfluramida (6) & 92,22 & 100,00 & 22,22 & 66,67 & 77,78 & 88,89 & 77,78 \\
Sulfluramida (8) & 93,33 & 0,00 & 11,11 & 66,67 & 77,78 & 100,00 & 100,00 \\
Sulfluramida (10) & 93,33 & 0,00 & 11,11 & 88,89 & 77,78 & 88,89 & 88,89 \\
Clorpirifós A (10) & 16,67 & 0,00 & 11,11 & 0,00 & 11,11 & 11,11 & 0,00 \\
Clorpirifós A (15) & 25,00 & 22,22 & 22,22 & 33,33 & 22,22 & 11,11 & 11,11 \\
Clorpirifós A (20) & 16,67 & 22,22 & 44,44 & 33,33 & 33,33 & 33,33 & 22,22 \\
Clorpirifós B (10) & 61,11 & 44,44 & 22,22 & 33,33 & 44,44 & 44,44 & 44,44 \\
Clorpirifós B (15) & 47,22 & 11,11 & 22,22 & 55,56 & 33,33 & 44,44 & 44,44 \\
Clorpirifós B (20) & 25,00 & 33,33 & 55,56 & 44,44 & 11,11 & 0,00 & 11,11 \\
Clorpiritós C (5) & 31,11 & 44,44 & 33,33 & 33,33 & 22,22 & 22,22 & 22,22 \\
Clorpirifós C (10) & 24,44 & 44,44 & 33,33 & 22,22 & 22,22 & 22,22 & 22,22 \\
Clorpirifós C (15) & 13,33 & 66,67 & 55,56 & 66,67 & 22,22 & 22,22 & 22,22 \\
\hline
\end{tabular}

após a aplicação da isca, reduzindo o tempo de conclusão sobre sua efíciência, possibilitando uma nova intervenção mais rapidamente e evitando maiores danos que poderiam ser causados pelos formigueiros sobreviventes desta espécie.

Verificou-se uma redução no número de olheiros $/ \mathrm{m}^{2}$ por formigueiro com o aumento da área dos mesmos (Fig.1).
Isso é muito importante quando se utiliza o método de dosagem única por olheiro para o controle de formigas cortadeiras. Assim, formigueiros menores receberão uma quantidade de isca muito próxima da dosagem recomendada por metro quadrado, produzindo boa eficiência no combate, enquanto formigueiros maiores receberão uma sub-dosagem de isca, comprometendo

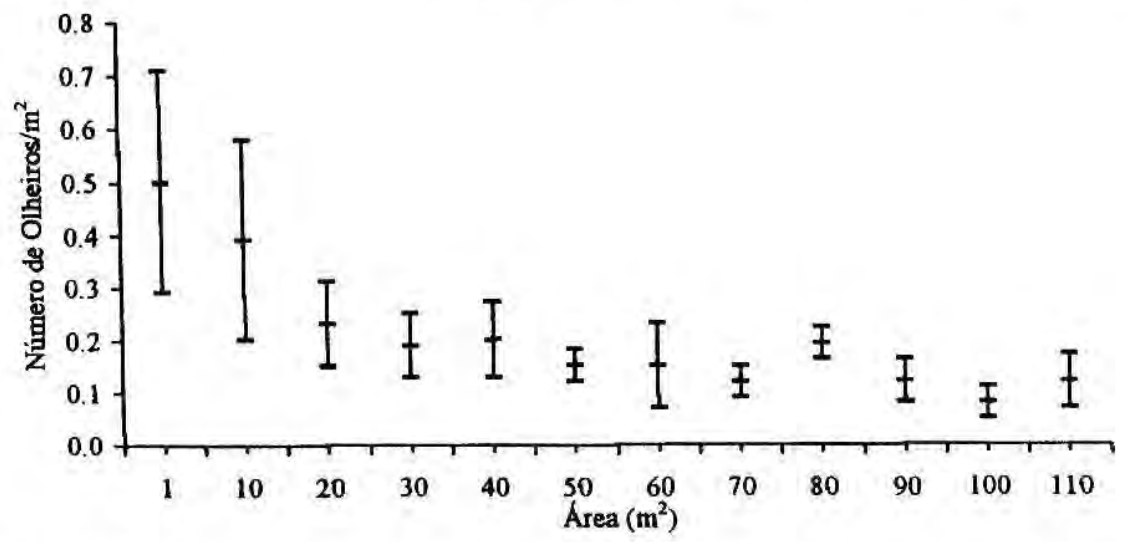

Figura 1 - Relação entre a área $\left(\mathrm{m}^{2}\right)$ e o número de olheiros por metro quadrado de formigueiro de Atta sexdens sexdens (Hymenoptera: Formicidae). Almeirim, Pará. 
sua eficiência. Esses dados são semelhantes aos de TORRES et al. (1995), que verificaram, para $A$. laevigata, uma redução entre $18,98 \%$ e $44,98 \%$ na quantidade de isca aplicada em formigueiros maiores quando se utilizou o método de dosagem única em comparação ao de dosagem por área de formigueiro. Desta forma, recomenda-se um aumento gradativo da quantidade de isca por olheiro com o aumento da área do formigueiro, quando se utilizar a metodologia de dosagem única para o controle de A. sexdens sexdens na região de Almeirim..

\section{Bibliografia Citada}

CAMERON, R.S. 1990. Potential baits for control of the Texas leaf cutting ant, Atta texana (Hymenoptera: Formicidae). In: Vander Meer, R.K.; JAFFÉ, K.; CEDENO, A. eds. Applied_myrmecology a world perspective, p628-637.

CAETANO, F.H.; PACHECO, P.; COSTACOELHO, L.C. 1993. Verificação da ação do sulfluramid-GX-071HB, isca granulada formicida sobre colônias de Acromyrmex aspersus (Hymenoptera: Formicidae). In: Anais do $14^{\circ}$ Congresso Brasileiro de Entomologia, Sociedade Entomológica do Brasil, Piracicaba, São Paulo, p517.

COUTO, L.; ZANUNCIO, J.C.; ALVES, J.E.M.; CAMPINHOS, E.; SORESINI, L.; VARGAS, J.A. 1977. Avaliação de eficiência de controle de Atta sexdens rubropilosa através do sistema de termonebulização, na região de Aracruz, ES. Revista Árvore, 1(1):9-16.

DELLA LUCIA, T.M.C. \& VILELA, E.F. 1993. Métodos atuais de controle e perspectivas. IN: DELLA LUCIA , T.M.C. (ed.). As formigas cortadeiras. Viçosa. P 163-190.
FORTI, L.C; PINHÃO, M.S.A; YASSU, W.K. PRETTO, D.R.; NAGAMOTO, N.S. 1993. Pesquisas com sulfluramida, no período de 1989 a 1993, para o controle de Atta spp. In: Anais do IV International Symposium on Pest Ants - XI Encontro de Mirmecologia. Belo Horizonte, MG, p23.

LARANJEIRO, A.J. \& ZANUNCIO, J.C. 1994. Avaliação da isca à base de sulfluramida no controle de Atta sexdens rubropilosa pelo processo de dosagem única de aplicação de iscas formicidas. IPEF (48/49):144-152.

MARICONI, F.A.M. 1970. As saúvas, São Paulo, Agronômica Ceres, $167 \mathrm{p}$.

SANTOS, G.P.; GOMES, J.M; ZANUNCIO, J.C.; BRANDI, R.M. 1979. Controle de saúvas pelo sistema de termonebulização na região de Timóteo, MG. Brasil Florestal, 9(38):18-20.

TORRES, J.B.; ZANUNCIO, J.C.; GASPERAZZO, W.L.; ZANUNCIO, T.V. 1995. Número de olheiros ativos e área de terra solta: aferição de dosagens para formigueiros de Atta laevigata. In: Anais do $15^{\circ}$ Congresso Brasileiro de Entomologia, Sociedade Entomológica do Brasil, Caxambú, Minas Gerais, p545.

VANDER MEER, R.K.; LOFGREEN, C.S.; WILLIAMS, D.F, 1985. Fluoroaliphatic sulfones: A new class of delayed-action insecticides for control of Solenopsis invicta (Hymenoptera: Formicidae). Journal of Economic Entomology, 78: 1190-1197.

VANDER MEER, R.K.; LOFGREEN, C.S.; WIL LIAMS, D.F. 1986. Control of Solenopsis invicta with delayed-action fluorinated. Pesticide Science, 17: 449-455.

VILELA, E.F. 1986. Status of leaf-cutting ant control in forest plantations in Brazil. In: LOFGREEN, C.S. ; VANDER MEER, R.K. eds. Fire ants and leaf-cutting ants: biology and management. Boulder, Westview press, p399-408.

ZANUNCIO, J.C.; VILELA, E.F.; NOGUEIRA, S.B. 1980. Emprego de iscas granuladas e pós secos no controle de Atta laevigata, no município de Curvelo, MG. Revista Árvore, 4(2): 221-226. 
ZANUNCIO, J.C.; COUTO, L.; SANTOS, G.P.; ZANUNCIO, T.V. 1992. Eficiência da isca granulada à base de sulfluramid, no controle da formiga cortadeira Atta laevigata (F. Sinith, 1858) (Hymenoptera: Formicidae), Revista Árvore, 16(3): 357-361.

ZANUNCIO, J.C.; COUTO, L.; FAGUNDES, M.; ZANUNCIO, T.V. 1993a. Eficiência da isca Mirex-S (sulfluranid 0,3\%) no controle da formiga cortadeira Afta bisphuerica Forel (Hymenoptera: Formicidae). Revista Árvore, 17(1): 85-90.

ZANUNCIO, J.C.; LARANJEIRO, A.J.; SOUZA de, O.F. 1993b. Avaliaçâa da isca à base de sulfluramida no controle de Acromyrmex subterraneus molestans (Hymenoptera: Formicidae), na região de Aracruz, Espírito Santo. In: Anais do IV International Symposium on Pest Ants - XI Encontro de Mirmecologia. Belo Horizonte, MG, p103.
ZANUNCIO, J.C,; RODRIGUES, F.A.; FAGUNDES, M.; BORESTAINER, $S$. 1993c. Eficiência da isca Mirex-S (sulfluramid $0,3 \%$ ) no controle de Acromyrmex crassispinus (Hymenoptera: Formicidae). In: Resumos do $14^{\circ}$ Congresso Brasileiro de Entomologia, Sociedade Entomológica do Brasil, Piracicaba, São Paulo, p696.

ZANUNCIO, J.C.; SANTOS, G.P.; FIRME, J.D. 1993d. Avaliaçāo da eficiência da isca granulada Mirex-S (sulfluramida $0,3 \%$ ) em Atta sexdens rubropilosa Forel, 1908 (Hymenoptera: Formicidae). In: In: Anais do IV Intemational Symposium on Pest Ants-XI Encontro de Mirmecologia. Belo Horizonte, MG, p102.

ZANUNCIO, J.C; Z ZANUNCIO, T. :; SANTOS, G.P. 1993e. A contribuição da pesquisa, em entomologia florestal, para a reduçã̃o dos impactos ambientais dos reflorestamentos. Anais do I Simpósio Brasileiro de Pesquisa Florestal, Belo Horizonte, MG, p136-142. 